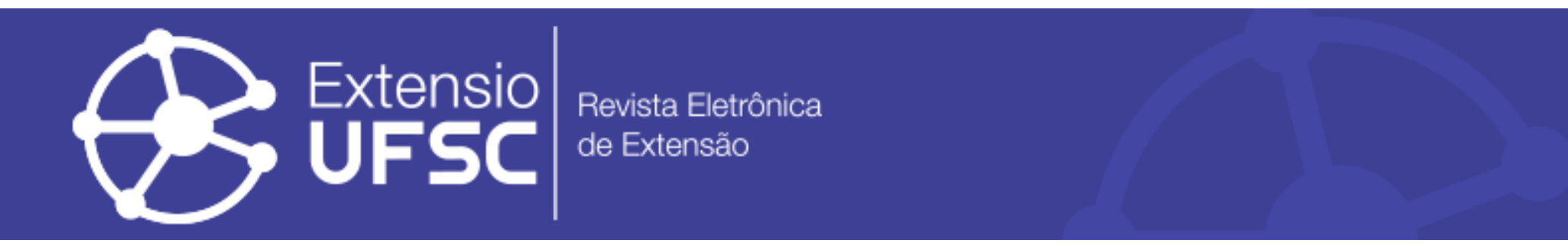

\title{
INSERÇÃO DE ESTUDANTES DE ODONTOLOGIA NO SUS: RELATO DE EXPERIÊNCIA
}

\author{
Pedro Vitali Kammer \\ Universidade Federal de Santa Catarina \\ pedrovkammer@gmail.com \\ Victória Hofstaetter \\ Universidade Federal de Santa Catarina \\ victoria.hofs@gmail.com \\ Evelise de Fátima Ferreira Sorgato \\ Universidade Federal de Santa Catarina \\ evelisesorgato@gmail.com \\ Mariana Steglich \\ Universidade Federal de Santa Catarina \\ marianasteglich@hotmail.com
}

Beatriz Sacchetti de Almeida Universidade Federal de Santa Catarina beatrizsacchettidealmeida@gmail.com

Maria Fernanda Silva da Luz Universidade Federal de Santa Catarina mariafernandasluz@hotmail.com

Juliana Santos de Simas Prefeitura Municipal de Florianópolis julianasimas@gmail.com

Maynara Schlickmann de Freitas Universidade Federal de Santa Catarina maynarafreitas@hotmail.com

\begin{abstract}
Resumo
As disciplinas do eixo de Saúde Pública, do curso de Odontologia da Universidade Federal de Santa Catarina (UFSC), têm como princípio formar indivíduos comprometidos com o Sistema Único de Saúde (SUS). Com a finalidade de capacitá-los a serem futuros transformadores da realidade atual, por meio do conhecimento sobre epidemiologia, planejamento e execução de políticas públicas. O objetivo deste trabalho é relatar a experiência dos alunos no planejamento e execução das atividades realizadas em conjunto com as Unidades Básicas de Saúde (UBSs), do município de Florianópolis. Os estudantes realizaram ações de promoção de saúde bucal e exames clínicos em escolares, além de participarem do planejamento e processo de trabalho das UBSs. A presença dos estudantes na rede pública de saúde contribui para capacitar futuros profissionais da saúde capazes de desenvolver práticas mais efetivas para as necessidades da comunidade e a serem futuros transformadores sociais da realidade do SUS.
\end{abstract}

Palavras-chave: Saúde Pública. Odontologia Comunitária. Sistema Único de Saúde.

\section{INSERTION OF DENTISTRY STUDENTS IN SUS: EXPERIENCE REPORT}

\begin{abstract}
The subject of Public Health, of the Federal University of Santa Catarina (UFSC) Dentistry course, aim to mold individuals committed to the work process of Brazilian's Unified Health System (SUS). With the purpose of capacitate them to, in the future; transform our current reality, through knowledge about epidemiology, planning, and execution of public regulations. This project aim to report the pupils' experiences in planning and performing of the activities developed in tandem with the Basic Health Unities (UBS) of Florianópolis. The academics brought up oral health promotion actions, performed clinical exams in primary school students, besides actively participate on the UBS' planning and work process. The academic's presence on the public health network can nurture future health professionals capable of cultivating practices that are more effective to fulfill the community's necessities and, therefore, remodel the social reality of the SUS. Keywords: Public Health. Community Dentistry. Unified Health System.
\end{abstract}

\section{INTERSECCIÓN DE ESTUDIANTES DE ODONTOLOGÍA EN SUS: RELATO DE EXPERIÊNCIA}

Resumen

Las disciplinas de Salud Pública, del curso de Odontología de la Universidad Federal de Santa Catarina (UFSC), tiene como principio formar individuos comprometidos con el proceso de trabajo del Sistema Único de Salud (SUS). Con la finalidad de darles la capacidad de ser futuros transformadores de la realidad actual, por medio del conocimiento sobre la epidemiologia, planteamiento y ejecución de políticas públicas. El objetivo de este trabajo es relatar la experiencia de los alumnos en el planteamiento y ejecucion de las actividades realizadas en conjunto con las Unidades Básicas de Salud (UBS) del municipio de Florianópolis. Los estudiantes realizaron actividades de promoción de salud bucal y la realización de exámenes clínicos en estudiantes, además de participar en el planteamiento y proceso del trabajo de las UBS. La presencia de los estudiantes en la red pública de salud puede promover futuros profesionales de Salud capaces de llevar a cabo prácticas más efectivas para las necesidades de la comunidad y ser futuros transformadores sociales de la realidad de SUS. Palavras clave: Salud Pública. Odontología Comunitaria. Sistema Único de Salud. 
Inserção de estudantes de Odontologia no SUS: relato de experiência

\section{INTRODUÇÃO}

Há diferentes definições de saúde, para Last (2007), saúde é o funcionamento integral da pessoa, sendo resultado do equilíbrio entre o ser humano e seu ambiente. Para Boorse (1987) saúde é ausência de doença e segundo Lalonde (1974), a saúde é um produto de várias variáveis, como estilo de vida, herança genética, meio ambiente e assistência médica. Por fim, a Organização Mundial da Saúde (OMS, 1946) conceitua saúde como um estado de completo bemestar físico, mental e social, e não apenas a ausência de enfermidades.

Visto a importância da saúde na vida do cidadão, o artigo 196 da Constituição Federal de 1988 diz que a saúde é direito de todos e dever do Estado. Assim, através de políticas sociais e econômicas é esperado que seja reduzido o risco de doenças e que se consiga o acesso universal e igualitário às ações e serviços para sua promoção, proteção e recuperação. Com base nisso, a Lei Orgânica da Saúde 8080/1990 (BRASIL, 1990) regulamenta o Sistema Único de Saúde (SUS).

A Unidade Básica de Saúde (UBS) se caracteriza como a principal forma de comunicação dos usuários com a Rede de Atenção à Saúde. A UBS é estabelecida próximo de onde a população reside, trabalha, estuda e vive e, dessa maneira, representa um papel crucial na garantia de acesso à comunidade a uma atenção à saúde de qualidade. As UBS têm em suas equipes médicos, enfermeiros, dentistas, auxiliares de enfermagem e pessoal de apoio técnico (ELIAS et. al., 2006).

Em 2005, o Ministério da Saúde criou um projeto chamado Programa Nacional de Reorientação da Formação Profissional em Saúde (Pró-Saúde), que tinha o objetivo de aproximar o graduando da atenção básica. Após a adesão desse programa foram criadas reformas curriculares, as quais de acordo com Freitas, Calvo e Lacerda (2012) são direcionadas à capacitação de profissionais efetivos e comprometidos com o SUS. A partir deste programa disciplinas voltadas à saúde pública foram instituídas nos cursos de Odontologia.

De acordo com as diretrizes estabelecidas pela Associação Brasileira de Ensino Odontológico (2002), o estágio supervisionado, permite ao aluno reconhecer a realidade social, o funcionamento dos serviços de saúde e o mercado de trabalho no qual será inserido. No caso presente, a experiência ocorre por meio da vivência do estudante na UBS.

Um estudo (ALMEIDA, 2012) realizado entre os anos de 2009 e 2010 em uma UBS em Fortaleza, concluiu-se que há uma contribuição positiva na inserção do aluno de graduação na UBS, pois os estudantes tornam-se conhecedores e possíveis modificadores da realidade. Os graduandos aprendem a trabalhar com a humanização, cuidado e capacitação do serviço, o que 
Inserção de estudantes de Odontologia no SUS: relato de experiência

contribuiu para sua formação como profissionais críticos, com consciência e capacidade para desenvolver ações voltadas para a prevenção, promoção e educação em saúde.

As disciplinas do eixo de Saúde Pública, implementadas no currículo do curso de Odontologia da UFSC, durante oito fases, tem como principal objetivo capacitar o estudante a compreender o processo de trabalho nas UBS, além de promover a vivência na rotina da rede pública de saúde.

Com objetivo de capacitar os alunos a compreender o processo de trabalho do cirurgião dentista na Atenção Básica do SUS, a disciplina baseia-se na Estratégia de Saúde da Família (ESF) para desenvolver ações nas quais os alunos, em parceria com a prefeitura municipal de Florianópolis, realizam atividades como: promoção de saúde bucal coletiva; educação e prevenção em saúde bucal em escolas e creches; levantamento de necessidades de tratamento odontológico; grupos de atenção primária e visitas domiciliares.

Diante disso, o objetivo desse trabalho é relatar as atividades desempenhadas pelos alunos de Odontologia da Universidade Federal de Santa Catarina (UFSC) em conjunto com algumas UBS da cidade de Florianópolis/SC durante a graduação.

\section{MÉTODOS}


Inserção de estudantes de Odontologia no SUS: relato de experiência

Durante os quatro anos das disciplinas, o conteúdo programático permeia o estudo de conceitos sobre saúde coletiva, epidemiologia e planejamento em saúde, e avaliação das estratégias de políticas públicas para promoção e estruturação da Atenção Básica em Saúde. O intuito é promover a inserção dos alunos no funcionamento do SUS, por meio da participação de reuniões de planejamentos, da ESF, dos Conselhos Municipais de Saúde e outros, gerando conhecimento da realidade política e socioeconômica por trás das estratégias elaboradas para a população-alvo de cada região.

Os alunos desenvolveram atividades dentro da UBS da quarta à oitava fase. Em cada semestre foram elaborados trabalhos de acordo com o plano de ensino das disciplinas. Uma das primeiras atividades realizadas, durante a disciplina de Planejamento e Programação em Saúde, foi a territorialização, a qual foi realizada em conjunto com os agentes de saúde, tendo como objetivo conhecer a região, a população e suas especificidades. Dados como frequência de uso dos serviços ofertados pela UBS, idade, doenças sistêmicas e facilidade no acesso a unidade foram investigados. Posteriormente, foi realizado um mapeamento, evidenciando as necessidades epidemiológicas de cada área para organizar os serviços de forma mais eficazes.

Durante a disciplina de Epidemiologia Aplicada à Saúde Bucal, outra atividade realizada pelos alunos foi a avaliação do Índice de Necessidade de Tratamento Odontológico (INTO). Foi realizado exame físico em crianças de 6 a 12 anos de escolas municipais, com o intuito de avaliar a saúde bucal e repassar os dados para a Prefeitura Municipal.

Durante o exame bucal foram registradas, de forma padronizada, informações como: número de cavidades (nesta classificação entram dentes permanentes e decíduos, e se exclui mancha branca); número de dentes para exodontia (identificando se o dente é permanente ou decíduo e excluindo-se os dentes com esfoliação normal); presença de sangramento gengival (observando se a criança tem sinais visíveis de inflamação, e perguntando-se se ao escovar os dentes sua gengiva sangra); se o examinado é respirador bucal (palato ogival, mordida aberta, lábio ressecado, entre outros), e a presença de cálculo dentário.

Presença de fraturas, mordida aberta, mordida cruzada, apinhamento, bruxismo, fístulas, e outras condições também foram registradas, ao final, foram somadas as necessidades de restaurações com as de exodontias, atribuindo um valor de 0 a 5 a cada paciente.

No 0 se enquadravam os indivíduos que não necessitavam de extrações/restaurações, 1 para os que possuíam de 1 a 3 necessidades de extrações/restaurações; 2 para os que possuíam de 4 a 8 necessidades; 3 para mais de 8 necessidades; 4 para os que possuem doença periodontal generalizada e 5 para necessidade de exodontias múltiplas. 
Inserção de estudantes de Odontologia no SUS: relato de experiência

Durante a disciplina de Odontologia em Saúde Coletiva, foram elaboradas atividades lúdicas desenvolvidas para alunos de Núcleos de Educação Infantil (NEI) e escolas municipais da Prefeitura de Florianópolis, abrangendo crianças de 5 a 8 anos. Essas atividades visaram a promoção de uma alimentação saudável e menos cariogênica, escovação dental, uso de fio dental e escovação supervisionada. Em algumas escolas foi realizado Tratamentos Restauradores Atraumáticos (ART) para crianças que necessitavam de tratamento restaurador, e possuíam a permissão escrita dos pais ou responsáveis. O ART consiste em um procedimento que não utiliza anestesia e instrumentos rotatórios, e tem como objetivo frear o processo de desmineralização da lesão cariosa em dentes decíduos e permanentes. Para realizar esse procedimento foram utilizadas curetas de dentina, espelho clínico, espátula e ionômero de vidro restaurador, além de Equipamentos de Proteção Individual (EPI) e gazes estéreis. Os atendimentos foram realizados nas escolas em uma sala com boa iluminação.

Posteriormente, os estudantes foram inseridos nas UBSs para vivenciar o funcionamento diário na disciplina de Processo de Trabalho na Atenção Primária. Os alunos ficaram em diferentes áreas de atendimento e realizaram rodízio. $\mathrm{Na}$ recepção, auxiliaram nas orientações das necessidades dos usuários, além do agendamento de consultas e exames. Na farmácia, assistiram a distribuição dos fármacos pelos responsáveis, foram instruídos sobre a correta forma de prescrever medicamentos para serem retirados no SUS e aprenderam sobre o gerenciamento dos medicamentos disponíveis. Já no acolhimento, os estudantes recepcionaram os usuários e, juntamente com o profissional da UBS realizaram anamneses e exames físicos iniciais com o objetivo de direcioná-los ao serviço necessário; acompanharam, na sala de procedimentos, aplicações de injeções e também curativos.

Além disso, presenciaram procedimentos de vacinação e atendimento odontológico com o Cirurgião-dentista da UBS. Em conjunto com os professores e dentistas preceptores, os estudantes desenvolveram ações para auxiliar na diminuição das necessidades dos usuários, detectadas durante processo de trabalho em diferentes áreas.

Diferentes assuntos foram apresentados aos usuários da UBS por meio de grupos. De modo geral, esses grupos buscaram instruir quanto à saúde bucal e geral. Foi realizado um grupo de bebês, onde as mães receberam instruções sobre escovação, erupção dos dentes decíduos, uso de chupeta, amamentação e também puderam tirar suas dúvidas relacionadas à saúde bucal de seus filhos.

No grupo de saúde bucal para idosos foi explicado como realizar a higienização da prótese, seu manuseio e os cuidados que devem ser tomados, principalmente caso venha a ocorrer fratura delas. Outros assuntos abordados foram as patologias mais comuns do 
Inserção de estudantes de Odontologia no SUS: relato de experiência

envelhecimento, além do câncer de boca e do autoexame bucal. Foram apresentadas imagens das principais lesões encontradas na boca e suas regiões mais frequentes.

Foi formado também um grupo para indivíduos tabagistas, que contou com o apoio de uma estudante de Psicologia. O objetivo desse grupo foi auxiliar na cessação do tabagismo, mostrar os aspectos negativos do seu consumo, além de promover apoio durante o processo por meio de reuniões semanais.

Por serem uma forma de promoção e prevenção de saúde, essas ações levam informações significativas à população de modo informal. Por meio de conversas e métodos didáticos com profissionais e estudantes da área da saúde, os usuários receberam instruções com o objetivo de melhorar sua própria qualidade de vida.

\section{RESULTADOS}

Realizar atividades externas à Universidade, e principalmente, no sistema público de saúde é uma vivência que possibilita aos alunos visualizarem a vulnerabilidade do SUS, conhecendo as dificuldades estruturais e organizacionais encontradas no processo de trabalho pelas equipes de saúde (EMMI et al., 2017). Por meio das disciplinas do eixo de Saúde Pública do curso de Odontologia da UFSC, os alunos passam a conhecer essas realidades, e, com o embasamento teórico adquirido, se tornam possíveis modificadores desses cenários, passam a buscar o aprimoramento no planejamento de ações e promovem um atendimento mais humano para a comunidade. Cada ação é desenvolvida pelos graduandos, professores e dentistas das UBSs e são projetadas de acordo com as demandas de cada local. O planejamento do semestre é realizado em reuniões, onde são discutidas as necessidades da população, quais ações podem ser feitas para atendê-las e formulando os cronogramas de atividades.

Ao longo das disciplinas, os alunos são expostos a conceitos básicos na disciplina de Sociologia da Sáude, além de como foi processo de formação e importância do SUS no Brasil, na disciplina de Políticas e Sistemas de Saúde. Ao longo de quatro anos, são abordados conceitos de territorialização, epidemiologia, promoção de saúde, entre outros. Essas práticas foram inseridas de acordo com o Pró-Saúde, que tem como objetivo a realização de uma abordagem integral no processo saúde-doença, e com isso incentivar uma prática capaz de transformar os cenários das comunidades por meio da geração de conhecimento e prestação de serviços (BRASIL, 2002; PALMIER et al., 2012).

\section{TERRITORIALIZAÇÃO}


Inserção de estudantes de Odontologia no SUS: relato de experiência

A territorialização é utilizada na prática da saúde pública para que, por meio do mapeamento das áreas de abrangência da ESF, obtenham-se informações referentes à qualidade de vida e necessidades da população para elaboração de estratégias em saúde (GONDIM et al., 2008). Essa conduta tem como objetivo avaliar a população pertencente a cada área, além de executar um levantamento das principais necessidades da comunidade. Isso possibilita a realização de estratégias mais eficazes, criadas pela ESF, para sanar as demandas dos usuários, seguindo os princípios norteadores do SUS, tais como equidade, integralidade e universalização. Por conta disso, a estruturação dos serviços de saúde tem um papel importante na atenuação das desigualdades, já que a busca por equidade na oferta desses serviços está vinculada ao desenvolvimento regional, gerando resultados positivos para a sociedade brasileira (GADELHA et al., 2011). A inserção dos alunos no auxílio deste levantamento é de suma importância, pois permite o conhecimento das demandas do usuário, além de introduzir noções sobre planejamento das ações da UBS. O conhecimento das necessidades especificas de cada área é essencial, pois os serviços ofertados pela UBS devem ser adaptados a cada população, e não o contrário.

\section{GRUPOS}

Em uma atuação que rompe com as barreiras das paredes do consultório odontológico, os grupos populacionais se constituem como uma forma de ações coletivas, que os cirurgiões dentistas e outros profissionais da ESF podem desenvolver. Essas atividades são mais frequentemente realizadas em forma de palestras e rodas de conversas (ALMEIDA; FERREIRA, 2008). Mobilizar diversas áreas e abordar tópicos relacionados à prevenção e promoção da saúde bucal e sistêmica, de maneira multidisciplinar, é essencial para envolver os participantes e integrar a equipe da ESF.

Durante o desenvolvimento dos grupos, foi observada a participação dos usuários quanto à compreensão das orientações passadas. No grupo de idosos, foi evidente o interesse durante as instruções para higienização da prótese e o autoexame bucal. A integração com outros profissionais, as atividades em grupos, assuntos relevantes para a comunidade e fora do consultório são fatores que podem colaborar para o bom desempenho dessas atividades. Esses encontros proporcionaram maior desenvolvimento pessoal dos estudantes, uma vez que é 
Inserção de estudantes de Odontologia no SUS: relato de experiência

necessário o domínio do conteúdo e a colaboração com outras áreas, como Psicologia e Enfermagem, além de aproximá-los aos usuários da UBS e de suas realidades.

\section{CONSELHO MUNICIPAL}

Segundo a Secretaria Municipal de Saúde de Florianópolis, o Conselho Municipal de Saúde de Florianópolis foi instituído em 1º de novembro de 1989 pela Lei Municipal nº 3291/89 (FLORIANÓPOLIS, 1989) e, tem por objetivo, a criação de estratégias, o controle e a fiscalização da execução de políticas de saúde. A plenária, que é composta por representantes das entidades-membro dos segmentos dos usuários, governo, prestadores de serviços da área da saúde e profissionais de saúde, se reúne mensalmente na última terça-feira de cada mês. Os alunos participaram de uma reunião do Conselho Municipal de Saúde de Florianópolis, realizada no Centro de Saúde da Trindade, onde foram discutidas questões como a falta de segurança e o assédio contra os funcionários do Centro de Saúde do Rio Vermelho, a construção de uma nova unidade, fatores relacionados à dengue no município e também a falta de atendimento odontológico em uma das Unidades de Pronto Atendimento. As pautas dessas reuniões são questões importantes para o sistema de saúde do município, que busca mais qualidade para seus funcionários, usuários e gestores.

\section{SAÚDE BUCAL EM ESCOLAS}

O INTO é um instrumento utilizado para o planejamento das atividades de saúde bucal, pois se trata de um método de vigilância epidemiológica que contribui para que os serviços odontológicos sejam oferecidos com equidade, colaborando com o agendamento de consultas individuais e a orientação na participação de procedimentos coletivos (WERNECK et al., 2011). A realização do INTO em escolares da rede pública de ensino tem sido uma atividade muito agregadora para o município de Florianópolis, assim como para os alunos da graduação em Odontologia da UFSC. Isso porque o atendimento aprimora ainda mais a técnica do aluno, tal como gera dados importantes para que a prefeitura consiga realizar atividades de promoção de saúde e atenda essa população alvo. Essa atividade realizada pelos alunos é contínua e abrange várias escolas do município.

Já o ART é uma estratégia de ação importante dentro das escolas, pois há uma grande prevalência de cárie em dentes decíduos, que são perdidos precocemente gerando problemas futuros (CARVALHO et al., 2013). Por ser uma técnica restauradora atraumática, simples, de 
Inserção de estudantes de Odontologia no SUS: relato de experiência

baixo custo, a qual é feita fora dos limites do consultório odontológico (SILVESTRE; MARTINS; SILVA, 2010), pode ser aproveitado de forma a promover saúde para a consolidação de comportamentos saudáveis e prevenção da doença cárie, que comumente afeta os tecidos dentais. Essa técnica proporciona aos estudantes aprimoramento da técnica, interrompe o desenvolvimento da atividade da carie, e é facilitada por não haver necessidade de instrumentos rotatórios, podendo ser executada em diversos lugares onde os consultórios odontológicos são distantes.

\section{CONSIDERAÇÕES FINAIS}

A presença dos estudantes nas UBSs promove a vivência dos estudantes de graduação na realidade do SUS. As disciplinas de IC cumprem com o objetivo de tornar os alunos mais capazes de atuar na rede pública, pois ao longo dos quatro anos diversas atividades preparam os estudantes a fim de capacitá-los para as diversas responsabilidades de um bom profissional.

Desde o planejamento até a execução das atividades os alunos desenvolvem atividades para transformar a realidade social dos usuários de cada UBS. O objetivo é promover não só a saúde bucal, mas também a saúde sistêmica, baseando-se em todo o conteúdo teórico disponibilizado pelas disciplinas, sendo modificadores da realidade ao promover uma atenção primária aos usuários do SUS mais humana e efetiva.

\section{REFERÊNCIAS}

ALMEIDA, F. C. M. et al. Avaliação da inserção do estudante na unidade básica de saúde: visão do usuário. Rev. bras. educ. méd, v. 36, n. 1, supl. 1, p. 33-39, 2012.

ALMEIDA, Gilmara Celli Maia de; FERREIRA, Maria Ângela Fernandes. Saúde bucal no contexto do Programa Saúde da Família: práticas de prevenção orientadas ao indivíduo e ao coletivo. Cad. Saúde Pública, Rio de Janeiro, v. 24, n. 9, p. 2131-2140, Sep. 2008

BOORSE, C.; VAN DE VEER, D.; REGAN, T. Health care ethics: An introduction. 1987.

BRASIL. Lei Orgânica de Saúde $\mathrm{n}^{\circ}$. 8.080, de 19 de setembro de 1990: Dispõe sobre as condições para a promoção, proteção e recuperação de saúde, a organização e o funcionamento dos serviços correspondentes e de outras providências. Diário Oficial da União, v. 1, 1990.

BRASIL. CNE Conselho Nacional de Educação. Câmara de Educação Superior. Resolução CNE-CES 3, de 19/02/2002 institui as Diretrizes Curriculares Nacionais do Curso de Graduação em Odontologia. Diário Oficial da União, 2002. 
Inserção de estudantes de Odontologia no SUS: relato de experiência

CARVALHO, T. H. L. et al. Estratégias de promoção de saúde para crianças em idade préescolar do município de Patos-PB. Revista Odontologia da Unesp, São Paulo, v. 42, n. 6, p. 426-431, 2013.

BRASIL. Constituição (1988). Constituição da República Federativa do Brasil. Brasília, DF: Senado Federal: Centro Gráfico, 1988.

ELIAS, Paulo Eduardo et al. Atenção Básica em Saúde: comparação entre PSF e UBS por estrato de exclusão social no município de São Paulo. Ciência \& Saúde Coletiva, v. 11, p. 633-641, 2006.

EMMI, D. T.; SILVA, D. M. C. d.; BARROSO, R. F. F. Experiência do ensino integrado ao serviço para formação em Saúde: percepção de alunos e egressos de Odontologia. InterfaceComunicação, Saúde, Educação, v. 22, p. 223-236, 2017.

FLORIANÓPOLIS. Lei n. 3291/89, de 01 de nov. de 1989. CRIA E DEFINE ATRIBUIÇÕES AO CONSELHO MUNICIPAL DE SAÚDE, Florianópolis, SC, nov 1989.

FREITAS, S. F. T. d.; CALVO, M. C. M.; LACERDA, J. T. d. Saúde coletiva e novas diretrizes curriculares em odontologia: uma proposta para graduação. Trab. educ. saúde, v. 10, n. 2, p. 223-234, 2012.

GADELHA, C. A. G. et al. Saúde e territorialização na perspectiva do desenvolvimento. Ciência \& Saúde Coletiva, v. 16, p. 3003-3016, 2011.

GONDIM, Grácia Maria de Miranda et al. O território da saúde: a organização do sistema de saúde e a territorialização. Território, ambiente e saúde. Rio de Janeiro: Editora Fiocruz, p. 237 $255,2008$.

LALONDE, M. A New Perspective on the Health of Canadians: A Working Document; Nouvelle Perspective De La Sante Des Canadiens. 1974.

LAST, J. M. A dictionary of public health. Oxford University Press, USA, 2007. ISBN 0195160908.

ODONTOLÓGICO, A. B. d. E. Diretrizes da ABENO para a definição do estágio supervisionado nos cursos de Odontologia. Revista da ABENO, v. 2, n. 1, 2002.

ORGANiZATION, W. H. Constituição da Organização Mundial da Saúde (OMS/WHO). 1946.

PALMIER, A. C. et al. Inserção do aluno de Odontologia no SUS: Contribuições do Pró-Saúde. Rev. bras. educ. méd, v. 36, n. 1 Supl 2, p. 152-157, 2012.

SILVESTRE, J. A. C.; MARTINS, P; SILVA, J. R. V. O tratamento restaurador atraumático da cárie dental como estratégia de prevenção e promoção de saúde da família. SANARE, Sobral, v. 9 , n. 2, p. 81-85, jul. 2010.

WERNECK, M. A. F., et al. Emprego do Índice de Necessidade de Tratamento Odontológico (INTO) na organização do acesso aos serviços de saúde bucal na UBS 
Inserção de estudantes de Odontologia no SUS: relato de experiência

Santa Rita de Euxenita, em Sabinópolis-MG. Apresentação de Trabalho: COBRAPO Congresso Brasileiro de Atenção Primária em Odontologia. 2011.

Recebido em: 18/01/2019

Aceito em: 19/11/2019 